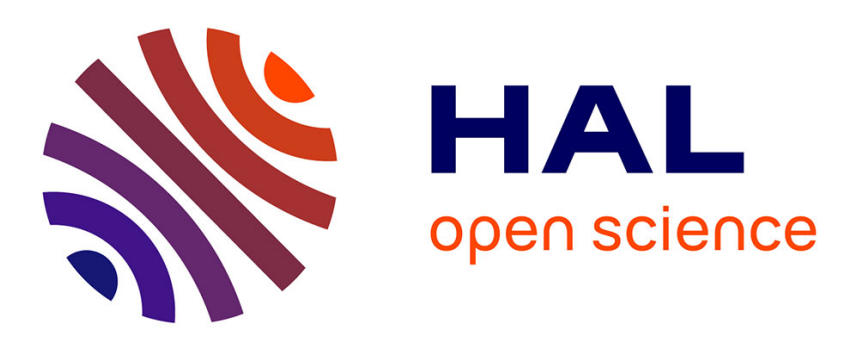

\title{
Structural heterogeneities at the origin of acoustic and transport anomalies in glycerol glass-former
}

\author{
Rémi Busselez, Thomas Pezeril, Vitali Goussev
}

\section{To cite this version:}

Rémi Busselez, Thomas Pezeril, Vitali Goussev. Structural heterogeneities at the origin of acoustic and transport anomalies in glycerol glass-former. Journal of Chemical Physics, 2014, 140 (23), 10.1063/1.4883504 . hal-01906157

\section{HAL Id: hal-01906157 \\ https://hal.science/hal-01906157}

Submitted on 6 Nov 2018

HAL is a multi-disciplinary open access archive for the deposit and dissemination of scientific research documents, whether they are published or not. The documents may come from teaching and research institutions in France or abroad, or from public or private research centers.
L'archive ouverte pluridisciplinaire HAL, est destinée au dépôt et à la diffusion de documents scientifiques de niveau recherche, publiés ou non, émanant des établissements d'enseignement et de recherche français ou étrangers, des laboratoires publics ou privés. 


\section{Structural heterogeneities at the origin of acoustic and transport anomalies in glycerol glass-former}

Rémi Busselez, Thomas Pezeril, and Vitalyi E. Gusev

Citation: The Journal of Chemical Physics 140, 234505 (2014); doi: 10.1063/1.4883504

View online: https://doi.org/10.1063/1.4883504

View Table of Contents: http://aip.scitation.org/toc/jcp/140/23

Published by the American Institute of Physics

\section{Articles you may be interested in}

Acoustic excitations in glassy sorbitol and their relation with the fragility and the boson peak

The Journal of Chemical Physics 137, 214502 (2012); 10.1063/1.4768955

Relaxation in glassforming liquids and amorphous solids

Journal of Applied Physics 88, 3113 (2000); 10.1063/1.1286035

Crystal nucleation as the ordering of multiple order parameters

The Journal of Chemical Physics 145, 211801 (2016); 10.1063/1.4962166

Liquid dynamics in partially crystalline glycerol

The Journal of Chemical Physics 146, 044502 (2017); 10.1063/1.4974831

Acoustical breakdown of materials by focusing of laser-generated Rayleigh surface waves

Applied Physics Letters 111, 031901 (2017); 10.1063/1.4993586

Laser-induced versus shock wave induced transformation of highly ordered pyrolytic graphite Applied Physics Letters 106, 161902 (2015); 10.1063/1.4918929

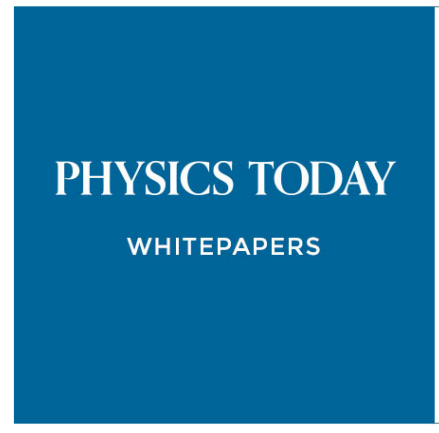

ADVANCED LIGHT CURE ADHESIVES

Take a closer look at what these environmentally friendly adhesive systems can do

\section{READ NOW}

PRESENTED BY Q. MASTERBOND" 


\title{
Structural heterogeneities at the origin of acoustic and transport anomalies in glycerol glass-former
}

\author{
Rémi Busselez, ${ }^{1, a)}$ Thomas Pezeril, ${ }^{1}$ and Vitalyi E. Gusev ${ }^{2}$ \\ ${ }^{1}$ Institut des Molécules et Matériaux du Mans UMR-CNRS 6283, Université du Maine, Le Mans, France \\ ${ }^{2}$ Laboratoire d'Acoustique de l'Université du Maine, UMR-CNRS 6613 Université du Maine, Le Mans, France
}

(Received 28 March 2014; accepted 3 June 2014; published online 18 June 2014)

\begin{abstract}
By means of large scale molecular dynamics simulations, we explore mesoscopic properties of prototypical glycerol glass-former above and below the glass transition. The model used, in excellent agreement with various experimental techniques, permits to carefully study the structure and the vibrational dynamics. We find that a medium range order is present in glycerol glass-former and arises from hydrogen bond network extension. The characteristic size of the structural heterogeneities is related to the anomalous properties of acoustic vibrations (Rayleigh scattering, "mode softening," and Boson Peak) in the glassy state. Finally the characteristic size of these heterogeneities, nearly constant in temperature, is also connected to the cross-over between structural relaxation and diffusion in liquid glycerol. () 2014 AIP Publishing LLC. [http://dx.doi.org/10.1063/1.4883504]
\end{abstract}

\section{INTRODUCTION}

In the glass transition field different theoretical approaches are stating that dynamical properties of glassforming systems have to be connected to the existence of heterogeneities in the supercooled liquid and glassy state. ${ }^{1-3}$ Some crucial aspects of the glass transition are still actually debated. In particular, the vibrational dynamics of glass forming liquids is still unclear, mainly due to the absence of experimental and numerical techniques covering at the same time a broad spectral and spatial domain. On one hand, Inelastic Neutron or X Ray scattering permits to cover a spatial range from Angstrom to nanometer, mainly in a narrow frequency band around THz. On the other hand Brillouin scattering permits to cover the $\mathrm{GHz}$ domain with a spatial resolution of hundred of nanometers. Hence a technical no man's land appears for the study of the spatially resolved dynamics in the $\mathrm{GHz}-\mathrm{THz}$ range in the nanometric domain. Unfortunately the nanometric domain corresponds to the expected size of heterogeneities in glass-forming liquids. Moreover, concerning the collective acoustic like excitations, experimental techniques are mainly able to study longitudinal polarization of acoustic waves and few techniques permit to reach the transverse component of the acoustic excitations ${ }^{4-8}$ near the $\mathrm{THz}$ region. Fortunately, the contribution of numerical techniques, facilitated by the increase of computers capacities, are able to shed light on this typical size range of few nanometers. Recently, large scale simulations upon model glass have shown that some aspects of acoustic anomalies can be related to a characteristic size in the glass. ${ }^{9,10}$ At the same time experimental works on real glasses have also shown the existence of a mesoscopic scale ${ }^{11-15}$ related to anomalies in the longitudinal acoustic excitations.

From another point of view, heterogeneities are also suspected to play a critical role in the transport mechanism and hence to modify the viscosity and density relaxation in liquid

\footnotetext{
a) remi.busselez@univ-lemans.fr
}

and supercooled state of glass-forming systems. ${ }^{16-23}$ Therefore two different length scales are relevant in order to understand the supercooled and glassy state of the glass-forming systems: a microscopic scale which is the fundamental aspect of original Mode Coupling Theory ${ }^{24}$ and a mesoscopic scale related to heterogeneities characteristic size. In this work we will demonstrate that in case of glycerol glass former such a mesoscopic scale responsible for the anomalous properties in acoustic excitations in glassy state is also responsible for the microscopic to macroscopic transport cross-over in liquid state and is intimately connected to structural heterogeneities associated to hydrogen bond network.

\section{MODELS AND METHODS}

In this paper, we performed molecular dynamics (MD) simulations of glycerol in liquid supercooled and glassy state. We used an all-atoms model of glycerol, ${ }^{25}$ which consists in an extension of the electrostatic and van der Waals point charge potential of Chelli et al. ${ }^{26,27}$ based on amber force field. Each of the 14 atomic force centers of the glycerol molecule was assigned to a partial charge to account for electrostatic interaction and a 6-12 Lennard-Jones (LJ) potential to account for repulsive and dispersive interactions. Because of the symmetry of the molecule, distinction is made between atoms located in the lateral positions and in the central position. This potential nicely reproduces both the dynamical and structural properties of the liquid phase..$^{20,25-28}$ MD simulations have been performed using the large-scale, massively parallel, molecular dynamics computer simulation code LAMMPS. ${ }^{29}$ Interatomic interactions were calculated using Lorentz-Berthelot mixing rules for the 6-12 LJ potential and PPPM method ${ }^{30}$ for long-range electrostatic interactions with cut-off radius of $r_{c u t}=12 \AA$. To investigate mesoscale range, we used different system sizes up to 4000 molecules. Using those sizes, lowest wavevector reachables are around $0.07 \AA^{-1}$. Simulations at $350 \mathrm{~K}$ and $310 \mathrm{~K}$ 
were first annealed during $10 \mathrm{~ns}$ in NPT canonical ensemble and then equilibrated in NVT ensemble using integration time of $0.5 \mathrm{fs}$. The simulation at $250 \mathrm{~K}$ was obtained starting from a configuration equilibrated at $350 \mathrm{~K}$, then cooled from $350 \mathrm{~K}$ to $250 \mathrm{~K}$ during a NPT run of $20 \mathrm{~ns}$ then equilibrated during another $20 \mathrm{~ns}$ run in NPT ensemble at constant temperature. After this process another additional NVT run of $20 \mathrm{~ns}$ was performed. We obtained the starting configuration at $150 \mathrm{~K}$ using $350 \mathrm{~K}$ equilibrated configuration (liquid state) and quenching during 40 ns simulation in isobaricisothermal ensemble. Then another additional $10 \mathrm{~ns}$ run in NVT ensemble was applied. This standard procedure led to mean density values of $1.29 \mathrm{~g} \mathrm{~cm}^{-3}$ at $150 \mathrm{~K}, 1.26 \mathrm{~g} \mathrm{~cm}^{-3}$ at $250 \mathrm{~K}, 1.22 \mathrm{~g} \mathrm{~cm}^{-3}$ at $310 \mathrm{~K}$, and $1.18 \mathrm{~g} \mathrm{~cm}^{-3}$ at $350 \mathrm{~K}$, giving less than $5 \%$ of relative error with reported experimental values. ${ }^{31,32}$ Starting from these configurations, production runs of $250 \mathrm{ps}$ were recorded in NVE ensemble using a time step of $0.5 \mathrm{fs}$, saving instantaneous positions and velocities every $0.01 \mathrm{ps}$.

The time correlation functions required to obtain the dynamic structure factor $S(Q, \omega)$, intermediate scattering function $S(Q, t)$, longitudinal and transverse current correlation function $C_{L / T}(Q, \omega)$ were computed from the instantaneous current functions:

$$
\begin{aligned}
\rho(\vec{q}, t) & =\frac{1}{\sqrt{N}} \sum_{i} w_{i} \exp \left(\imath \vec{q} \overrightarrow{r_{i}(t)}\right), \\
\overrightarrow{J_{L}(\vec{q}, t)} & =\frac{1}{\sqrt{N}} \sum_{i} m_{i}\left(\left(\overrightarrow{v_{i}(\vec{t})} \hat{q}\right) \hat{q}\right) \exp \left(\imath \vec{q} \overrightarrow{r_{i}(\vec{t})}\right), \\
\overrightarrow{J_{T}(\vec{q}, t)} & =\frac{1}{\sqrt{N}} \sum_{i} m_{i}\left(\hat{q} \otimes\left(\overrightarrow{v_{i}(\vec{t})} \otimes \hat{q}\right)\right) \exp \left(\imath \vec{q} \overrightarrow{r_{i}(\vec{t})}\right), \\
S(Q, t) & =\left\langle\rho(\vec{q}, t) \mid \rho^{*}(\vec{q}, 0)\right\rangle, \\
C_{L / T}(Q, \omega) & \left.\left.=\int\left\langle\overrightarrow{J_{L / T}(\vec{q}, t)}\right| \overrightarrow{J_{L / T}^{*}(\vec{q}, 0)}\right)\right\rangle e^{i \omega t} d t,
\end{aligned}
$$

where $Q$ stands for the wavevector modulus, $\vec{q}$ is the wavevector, $\hat{q}$ is the normalized wavevector, $v_{i}(t), r_{i}(t)$ are, respectively, the instantaneous velocity and position of atom $i$, and $m_{i}$ is the mass of atom $i$. During the analysis, we calculate two different series of correlation functions, one using all atoms (AA) in the system weighted by their corresponding mass, the second serie was calculated upon position and velocity of molecular center of mass (CoM). Using this bias, we coarse-grained the analysis and became able to separate the collective motions of molecules from the local motions. Functions expressed in frequency domain were obtained via Fourier transform of time dependent correlations. We used Hanning window with a width of $\Delta t \approx 100 \mathrm{ps}$, giving a resolution function of $10 \mathrm{GHz}$ in frequency domain. Density and current functions were averaged over independent directions of wavevector such $q=2 \pi\left(\frac{h}{L_{x}}, \frac{k}{L_{y}}, \frac{l}{L_{z}}\right)$, where $L_{x}, L_{y}$, and $L_{z}$ are dimensions of parallelepipedic box, giving lowest $\mathrm{q}$ of $0.07 \AA^{-1}$.

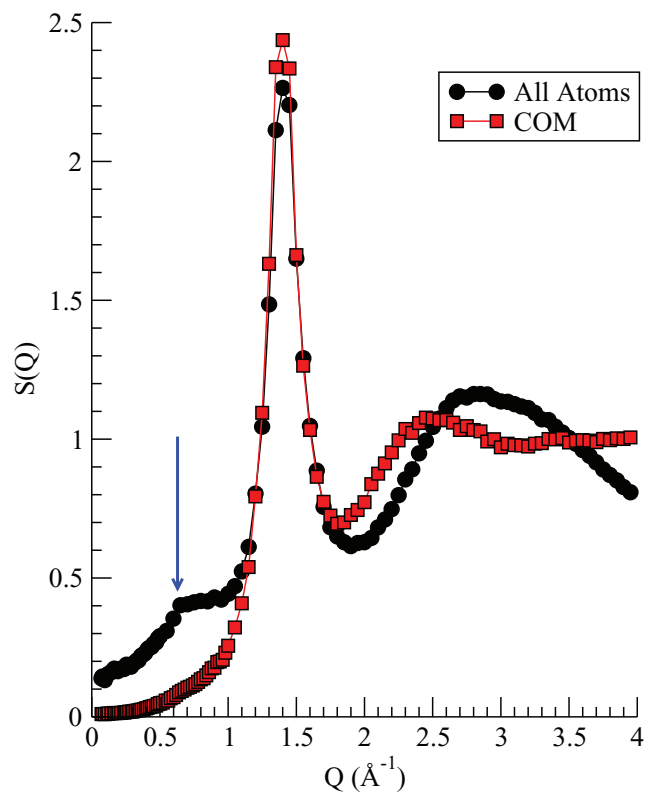

FIG. 1. Static structure factor at $350 \mathrm{~K}$. Black circles: static structure factor obtained from all-atom analysis and equal atom weighting. Red squares: structure factor obtained from center of mass analysis.

\section{RESULTS}

\section{A. Structure}

Information about liquid structure was obtained via static structure factor calculation. This magnitude was calculated using AA position and the molecular CoM (Figure 1). Looking to the atomic structure factor, the first sharp diffraction peak (FSDP) appears around $Q_{\max }=1.4 \AA^{-1}$, this value is in agreement with previous calculations obtained with different weighting coefficient (see Refs. 20, 25, and 28).

We can notice that the first sharp diffraction peak obtained from CoM positions (red squares), has the same location, width and has a similar height as the atomic structure factor. At these wavevectors, structure factor mainly reflects the intermolecular correlations (although intramolecular correlations exist for those wavevectors) ${ }^{33}$ and then explains the agreement between AA and CoM structure factor. In AA structure factor, a broad peak is present at $\mathrm{Q}=$ $3 \AA^{-1}$ and originates from intra and intermolecular correlations between closer atoms $(\approx 2 \AA$ in direct space). In the same time a small oscillation appears in the CoM S(Q) at $2.5 \AA^{-1}$ and originates from damped oscillations in intermolecular correlations.

Looking towards lower $\mathrm{Q}$ values, a shoulder is present around $0.7 \AA^{-1}$ in atomic structure factor. This shoulder has previously been reported by Blieck et al. ${ }^{25}$ on the same model and was interpreted as a pre-peak. A pre-peak located at low Q-range is generally related to the presence of preferential structure in liquids beyond the first atomic shell ${ }^{34-36}$ and then indicates a medium range order present in our system.

As the total structure factor is averaged over all the pair correlations in the system, it remains difficult to access from this magnitude a detailed view of the local structural order. To circumvent this problem, we also calculated the partial structure factor for the atoms belonging to the hydroxyl 


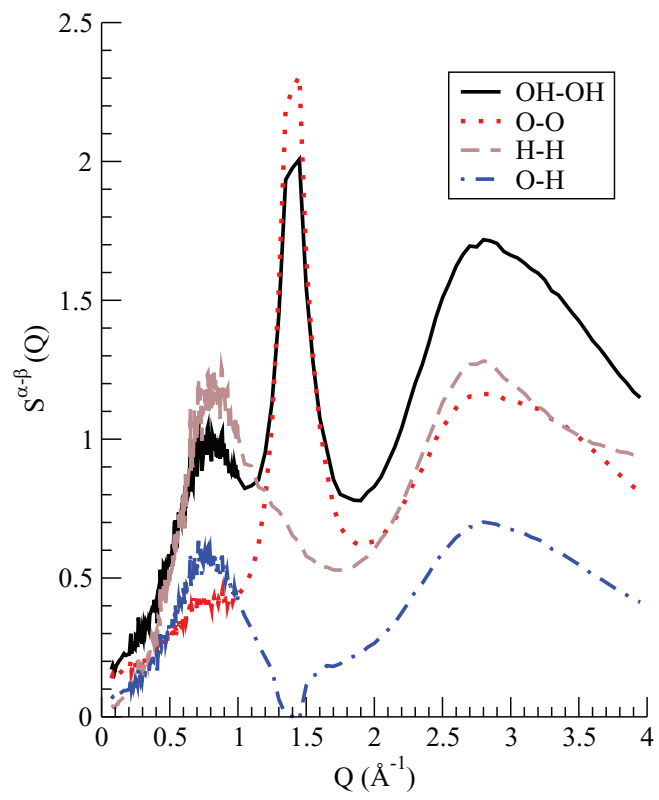

FIG. 2. Black lines: partial structure factor between hydroxyl group at $350 \mathrm{~K}$. Red dotted line: correlations between oxygen. Brown dashed line: correlations between hydrogen from hydroxyl groups. Blue line: crosscorrelations between oxygen and hydrogens from hydroxyl group.

groups. Results of this analysis are reported on Figure 2. Decomposition of hydroxyl structure factor leads to four different correlations: hydroxyl/hydroxyl correlations, oxygenoxygen correlations, hydrogen-hydrogen correlations, and finally cross-correlations between oxygen and hydrogen. Looking around the FSDP range (in the range $1-1.8 \AA^{-1}$ ), intensity for $(\mathrm{OH}) /(\mathrm{OH})$ correlations (black) is relatively high and comparable to the atomic structure factor in Figure 1. Contribution of $\mathrm{H} / \mathrm{H}$ and $\mathrm{O} / \mathrm{H}$ cross-correlations at those wavevectors is on the contrary very low and we can conclude straightforwardly that FSDP of hydroxyl groups is mainly given by the $\mathrm{O} / \mathrm{O}$ structure.

Looking to lower wavevectors and more precisely in the $0.5-1 \AA^{-1}$ region, we clearly see in the $\mathrm{OH} / \mathrm{OH}$ correlations a pre-peak located around $0.7 \AA^{-1}$. This pre-peak appears as a shoulder in the $\mathrm{O} / \mathrm{O}$ correlations as in atomic structure factor (Figure 1). $\mathrm{H} / \mathrm{H}$ correlations of alcohol groups show a prominent intensity rise around $0.7 \AA^{-1}$, indicating that the hydrogen of hydroxyl groups mainly dominates the intensity of the pre-peak. The wavevector range at which this structural organization arises $\left(\mathrm{Q} \approx 0.6-0.7 \AA^{-1}\right)$ indicates a characteristic distance of nanometric size and hence cannot be understood in terms of intramolecular organization. Thus we have to consider that molecules are associating through hydrogen bonds and form local structures separated with a typical nanometric distance. This picture is consistent with structural behavior of other molecular liquids showing a pre-peak ${ }^{35-38}$ or interacting via hydrogen bonding as neat alcohols ${ }^{39-41}$ giving rise to small clusters of hydrogen bonded molecules and thus tends to form a medium range order. In our case, pre-peak arises from hydroxyl group spatial organization and consequently should not appear in partial structure factor which does not integrate hydroxyl organization. This can be checked in the case of CoM structure factor, as CoM of glycerol molecule is located at the vicinity of the central carbon, its structure factor is not influenced by the hydroxyl position and hence no pre-peak or shoulder appears in CoM structure factor in Figure 1.

Although a medium range order was suspected in previous works, ${ }^{42,43}$ in some recent neutron scattering studies ${ }^{44,45}$ the shoulder towards low Q is absent. Despite the model used in our work remains compatible with neutron scattering experiments for $\mathrm{Q}$ values higher than $1 \AA^{-1}$ (Refs. 20 and 25) it seems to overestimate the low $\mathrm{Q}$ range. In fact an explanation of such discrepancy at low $\mathrm{Q}$ and agreement at higher $\mathrm{Q}$ might arise in the glycerol backbone conformation. The model used is based on the work of Chelli et al. ${ }^{26,27}$ where the conformations of glycerol backbone are mainly $\alpha \alpha(48 \%)$ and $\alpha \gamma$ $(46 \%)$, in qualitative disagreement with empirical potential structure refinement refinements of Ref. 44 where $\alpha \beta$ conformation is found, but close to ab initio calculations ${ }^{46}$ where the proportions are $70 \% \alpha \alpha$ and $27 \% \alpha \gamma$ conformers. It remains possible that the conformer proportions in our model induce slight changes in medium range order and finally conduce to the shoulder observed in low $\mathrm{Q}$ region. In the same manner, in neutron scattering experiments for 1-propano ${ }^{47}$ a shoulder at $Q=0.75 \AA$ appears in the structure factor at low temperature and is absent at high temperature. This low $\mathrm{Q}$ shoulder arises from a subtle balance in partial structure factor ponderation where small variations in the molecular conformation are related to the pre-peak apparition. Despite this overestimation in low $\mathrm{Q}$ intensity, all the analysis of experimental diffraction pattern $^{4-44}$ indicate the presence of correlation lengths up to $18 \AA$, signature of the existence of a medium range order in the liquid.

\section{B. Density correlations}

Structural relaxation can be studied through the density correlation function obtained in the time domain. As for the structure factor we calculated density-correlation for allatoms (using mass weighting), and for the center of mass. The normalized density correlator of the molecular CoM at $350 \mathrm{~K}, 310 \mathrm{~K}$, and $150 \mathrm{~K}$ is displayed in Figure 3 for 3 different wavevectors.

At high temperatures $(350 \mathrm{~K}$ and $310 \mathrm{~K})$ the coherent intermediate scattering functions show a standard behavior of liquids: at short time (below $2 \mathrm{ps}$ ) a first decay associated to vibrational modes, followed by along time decay, associated to the $\alpha$ relaxation regime. For liquid states ( $350 \mathrm{~K}$ and $310 \mathrm{~K}$ ) we analyzed the long-time relaxation of density correlations using the Kohlrausch-William-Watts (KWW) functional form

$$
S(Q, t)=A(Q) \exp \left(-\left(\frac{t}{\tau(Q)}\right)^{\beta(Q)}\right),
$$

(Figure 3 dashed curves). This stretched exponential law is a convenient empirical way to describe relaxation processes in glass-forming materials beyond the vibrational regime. Here $\tau(Q)$ stands for the wavevector dependence of coherent relaxation time, whereas $\beta(Q)$ reflects the wavevector dependence of the non-Debye character of the relaxation process. This expression is suitable for structural relaxation of glass-formers, as glycerol, in liquid and supercooled states. ${ }^{19,20,48,49}$ From 


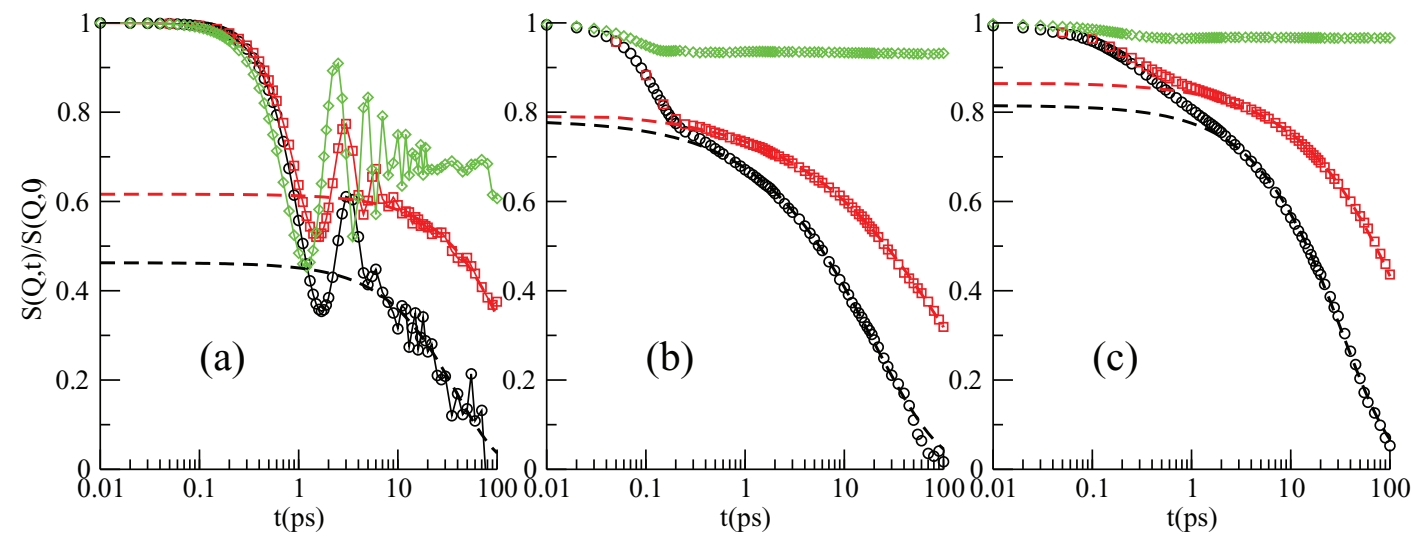

FIG. 3. From left to right: coherent intermediate scattering function of CoM at different wavevectors: $Q=0.08 \AA^{-1}$ (a), $Q=0.75 \AA^{-1}$ (b), and $1.5 \AA^{-1}$ (c). For each wavevector, we represent the intermediate scattering function at $350 \mathrm{~K}$ (black circles), $310 \mathrm{~K}$ (red squares), and $150 \mathrm{~K}$ (green diamonds).

this analysis, we obtained the collective mean relaxation time

$$
\langle\tau(Q)\rangle=\frac{\tau_{\mathrm{KWW}}(Q)}{\beta(Q)} \Gamma\left(\frac{1}{\beta(Q)}\right),
$$

where $\Gamma$ stands for Gamma function. The mean relaxation time is often used in KWW analysis in place of relaxation time due to the mutual dependency of $\beta$ and $\tau$ during the fit procedure. The $\beta$ values obtained during the fit procedure are around 0.6 at high $\mathrm{Q}$ values and progressively increase towards 0.85 at lowest $Q$ values in agreement with the experimental ones found at those wavelengths for self $19,20,48,49$ or collective relaxation. ${ }^{50}$ We report the Q-dependence of the coherent mean relaxation time on Figure 4 in the liquid state.

Relaxation times obtained using both molecular CoM analysis and AA reproduce the same global behavior for both temperatures studied, despite some differences at high wavevectors. At high $\mathrm{Q}$, collective relaxation times are fast and slowdown towards lower $\mathrm{Q}$ until a maximum located

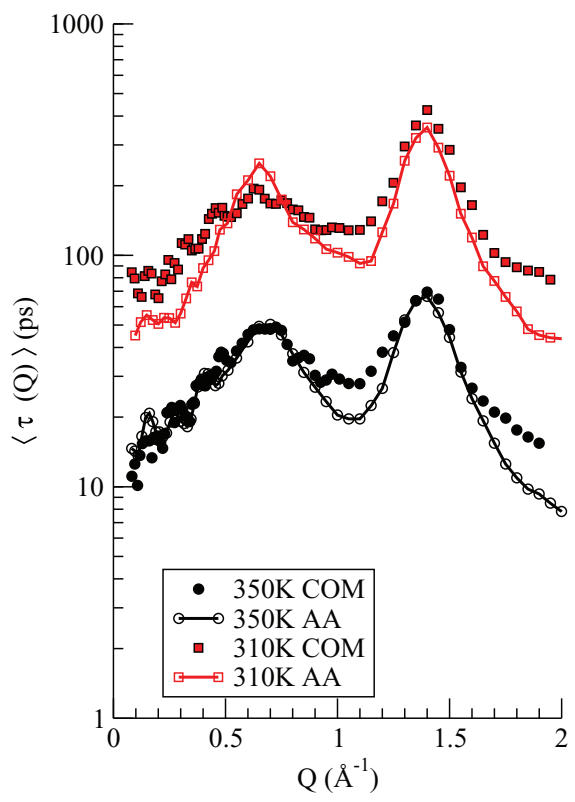

FIG. 4. Mean collective relaxation time at $350 \mathrm{~K}$ (black) and $310 \mathrm{~K}$ (red) for all-atoms (empty symbols and lines) and CoM (filled symbol) obtained from KWW analysis of coherent intermediate scattering function. at $1.4 \AA^{-1}$ where slow relaxation times are reached. Below this point, relaxation times decrease until a minimum at $\mathrm{Q}=1 \AA^{-1}$. Decreasing $\mathrm{Q}$, the relaxation times increase again to a second maximum located around $0.7 \AA^{-1}$. Then to lower $\mathrm{Q}$ values, relaxation times decrease. We would emphasize two observations, first collective relaxation times found at low Q (around pre-peak) are of the same order of magnitude as collective relaxation times around main peak and second CoM collective relaxation times are comparable to the all-atoms relaxation times, including the low-Q rise in relaxation times. Definitely two peaks are present in $\tau(Q)$, one located at $1.4 \AA^{-1}$ and the second located around $0.7 \AA^{-1}$. The first peak is general when studying collective dynamics of liquids and corresponds to the so-called De Gennes narrowing, ${ }^{51-53}$ this rise in collective relaxation times occurs in density-fluctuation at the cage scale and appears in both CoM and AA analysis at the first sharp diffraction peaks corresponding to the correlation between first neighbors at $Q_{p}$ $=1.4 \AA^{-1}$. The second peak is more intriguing and the microscopic explanation is not direct. We would first underline the location coincidence of the second maximum in collective density-fluctuation with the shoulder appearing in the static structure factor $S(Q)$ at $Q_{p p}=0.7 \AA^{-1}$ corresponding to structural correlation arising from medium range order of hydroxyl groups. However, S(Q) value for all-atoms at this wavevector is around 0.4 and hence a De Gennes narrowing effect cannot explain the sudden rise in the collective dynamics. The situation is even worse in the case of CoM, where structural relaxation times around $Q_{p p}=0.7 \AA^{-1}$ are still of the same order of magnitude as for the collective relaxation time obtained $Q_{p}=1.4 \AA^{-1}$, despite the very weak value of $S(Q)$ at low-Q, signature of the absence of structural correlations between the CoM at those length scale. The coherent relaxation times found in our study seem to agree well with a recently proposed ansatz ${ }^{16,23}$ connecting the local cage scale and mesoscopic dynamics via viscoelasticity of the liquid. This model implies a crossover between two different relaxation mechanisms of density fluctuations. This model is in agreement with previous numerical and experimental works on self dynamics of glycerol ${ }^{19,20}$ where at $\mathrm{Q}$ values around $0.5 \AA^{-1}$ a dynamic crossover appears in incoherent relaxation time from a non-Debye relaxation process to a Fickian 
diffusive process corresponding to hydrodynamic limit at low $\mathrm{Q}$ values.

\section{Current correlations}

In order to study the acoustic excitations, we have calculated both transverse and longitudinal current using all-atom calculations and CoM calculations. We calculated current correlations starting from the lowest wavevector accessible until the pseudo-Brillouin zone defined as the half value of the structural peak which in our case is located at $0.7 \AA^{-1}$. As we are interested in acoustic-like excitations represented by the low frequency part of the collective excitations spectra, we mainly focus on the CoM expressions of the current density. This kind of procedure is currently used in the acoustic-like excitations analysis of water. ${ }^{54,55}$ For glycerol, we checked that this bias keeps unmodified the low frequency part of the spectrum, comparing with the AA spectra. In Figure 5, we report longitudinal and transverse current spectra at $350 \mathrm{~K}$ for different $\mathrm{Q}$ values. At first sight, current density spectra can be represented as a narrow peak for longitudinal and transverse polarizations at low $\mathrm{Q}$. Towards greater $\mathrm{Q}$, current density spectra become broader despite a marked maximum. Clearly, the frequencies of maximum longitudinal excitations shift faster with $\mathrm{Q}$ than transverse excitations. In the Q-range presented in the following $\left(0.07\right.$ to $\left.0.7 \AA^{-1}\right)$, the longitudinal (L) and transverse (T) excitations have only one clear contribution. In order to extract the vibrational properties of liquid and glasses, we analyzed the current spectra in terms of simple damped harmonic oscillator (DHO) function.

$$
C_{L / T}(Q, \omega) \propto \frac{\omega^{2} \Gamma_{L / T}(Q)}{\left(\omega^{2}-\Omega_{\mathrm{L} / \mathrm{T}}(Q)^{2}\right)^{2}+\omega^{2} \Gamma_{L / T}(Q)^{2}} .
$$
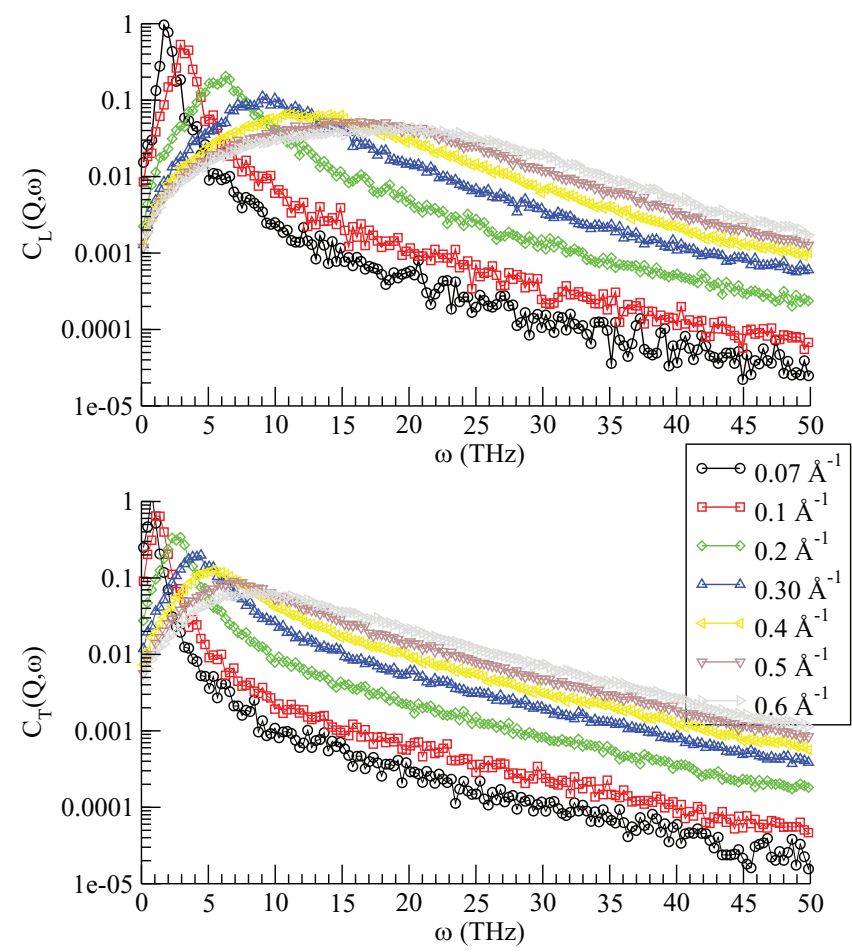

FIG. 5. Longitudinal (top) and transverse (down) current spectra of glycerol at $350 \mathrm{~K}$ for different $\mathrm{Q}$ values.
In the latter formula $\Omega(Q)$ represents the Brillouin pulsation for wavevector $\mathrm{Q}$ and $\Gamma(Q)$ represents the Brillouin linewidth associated to Q. In Figure 6, we present for each studied temperature the resonance and linewidth of Brillouin acoustic modes. The latter can be approximated by means of propagating quasiplane-waves while the mean free-path associated to acoustic excitation is greater than half the wavelength of the mode $\left(\ell(\omega) \gg \frac{\lambda(\omega)}{2}\right.$ following the denomination of Ref. (15). Rewriting this inequality in terms of Brillouin frequency (related to the wavelength of acoustic mode) and linewidth (related to the mean-free path of acoustic mode), we obtained the so-called Ioffe-Regel criterion given by $\Omega(Q)$ $\gg \pi \Gamma(Q)$ or $v(Q) \gg \frac{\Gamma(Q)}{2} .9,10,15,56-59$ Beyond this criterion, vibrations are no more propagating waves and hence the quantification of acoustic modes with wavevector quantum number loses its straightforward signification. ${ }^{56,60,61}$

In liquid state (Figs. 6(a) and 6(b), 350 K and $310 \mathrm{~K}$ ), we observed that $v$ for $\mathrm{L}$ modes evolves linearly in low-Q values up to $0.5 \AA^{-1}$. After that, frequency progressively flattens and decreases after $0.7 \AA^{-1}$ (not shown), the position of the pseudo-Brillouin zone of the system defined by half the first sharp diffraction peak $\left(Q_{p} / 2\right)$. In the same region, the linewidth of $\mathrm{L}$ excitations evolves following a power law with Q. Due to the linear evolution of frequency and power law evolution of linewidth, both curves are crossing each other when Ioffe-Regel criterion is reached around $Q_{L}^{I R}=0.2 \AA^{-1}$ at $350 \mathrm{~K}$ and $0.22 \AA^{-1}$ at $310 \mathrm{~K}$ for L modes. Hence, below this limit the longitudinal wave behaves as propagating quasiplane-wave and beyond this limit the acoustic excitations are localized modes and do not propagate.

For T modes, the situation is rather different: below 0.24 $\AA^{-1}$ at $350 \mathrm{~K}$ and $0.28 \AA^{-1}$ at $310 \mathrm{~K}$, a strict relation $v(Q)=$ $\Gamma(Q) / 2$ is followed. Thus half wavelength and mean-free path of the corresponding modes are identical, implying an evanescent nature of those modes and a non propagative character of large wavelength $\mathrm{T}$ modes in liquid state. This observation is an expected behavior of the liquid theory, directly connected to the inability of liquids to sustain shear strains. Towards higher $\mathrm{Q}$ and beyond IR criterion, linewidth is higher than mode frequency and is a clear indication of the T modes localization. Thus this limit indicates the crossover from evanescent $\mathrm{T}$ waves (which do not propagate in liquids) to diffusive or localized $\mathrm{T}$ waves (which do not propagate). However, both non-propagating characters of shear waves are of different natures and imply a cross-over from macroscopic (below $Q_{T}^{I R}$ ) to microscopic (above $Q_{T}^{I R}$ ) behavior.

Looking now to lower temperatures, in supercooled and glassy state (Figs. 6(c) and 6(d), respectively), mode frequencies $v(Q)$ for both polarizations have the same global behavior as in liquid state: a nearly linear evolution with $\mathrm{Q}$ followed by a progressive flattening when approaching the pseudo-Brillouin zone and finally a decrease of mode frequency approaching the pseudo-Brillouin zone (not shown). In the same time, Brillouin linewidth of both polarizations follows a power law in low Q part of the spectra, roughly until $0.5 \AA^{-1}$, after this point another law with smaller power appears. Values of half Brillouin linewidths $\Gamma(Q) / 2$ are clearly smaller than $v(Q)$ for $\mathrm{L}$ and $\mathrm{T}$ polarizations in the low $\mathrm{Q}$ range, implying that IR criterion is not reached. Consequently 

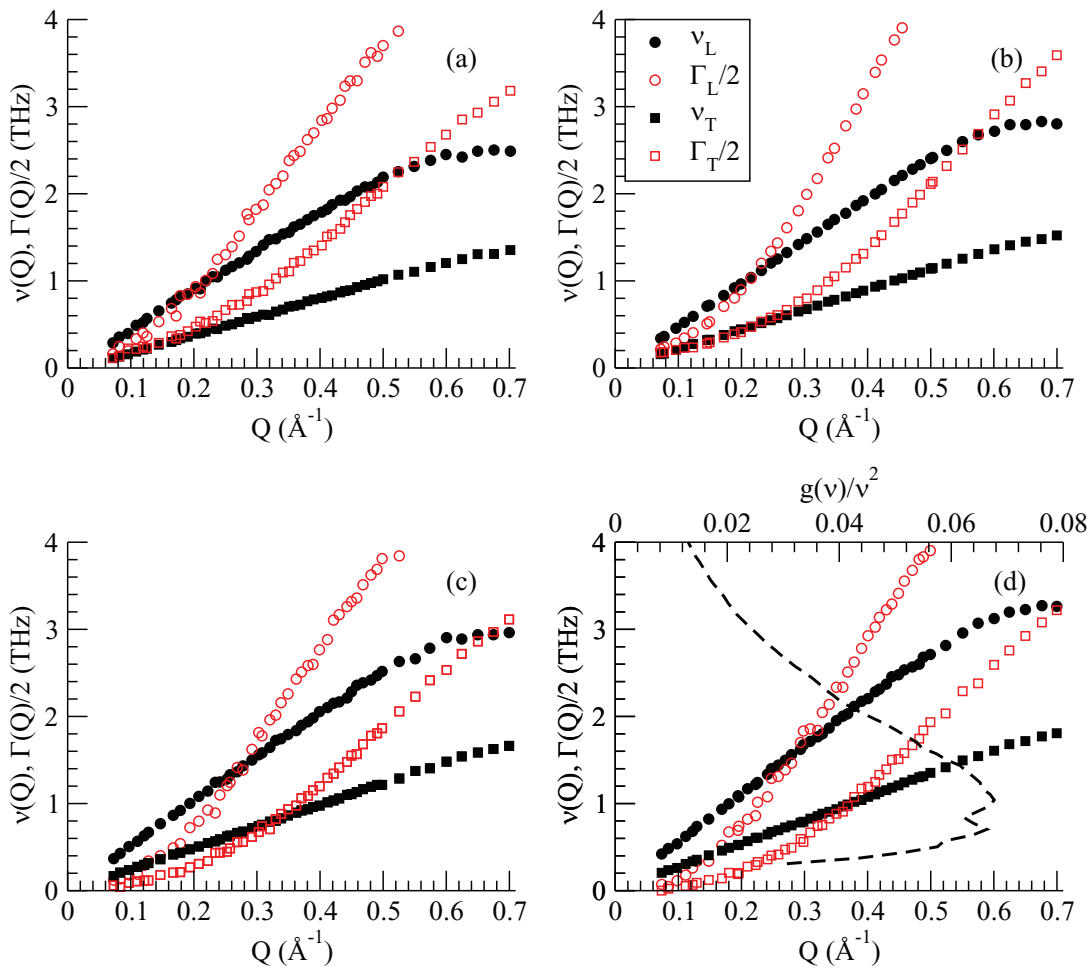

FIG. 6. $v$ and $\Gamma / 2$ (respectively, Black and Red) for longitudinal and transverse polarizations (respectively, circles and square) at four different temperatures: (a) $350 \mathrm{~K}$, (b) $310 \mathrm{~K}$ (c) $250 \mathrm{~K}$, and (d) $150 \mathrm{~K}$. In figure (d) we superimpose the reduced density of states calculated for this temperature and plotted for the same frequency range. Note the correspondence of Boson Peak with transverse Ioffe-Regel criterion.

the corresponding acoustic modes propagate in the system. We must emphasize that Ioffe-Regel criterions are not located to the exact same wavevector for $\mathrm{L}$ and $\mathrm{T}$ modes: at $250 \mathrm{~K}$ we find $\left(Q_{L}^{I R} \approx 0.25 \AA^{-1} ; Q_{T}^{I R} \approx 0.32 \AA^{-1}\right)$ at $150 \mathrm{~K}\left(Q_{L}^{I R}\right.$ $\left.=0.28 \AA^{-1} ; Q_{T}^{I R}=0.36 \AA^{-1}\right)$. At $150 \mathrm{~K}$ in glassy state (Figure 6(d)), we also represent the reduced density of states $\frac{g(v)}{v^{2}}$, calculated from CoM velocity correlations, for the same frequency range as analyzed modes. The reduced vibrational DOS presents a clear broad peak in the low frequency part. This peak in the reduced density of states is known as "density excess" above the Debye model and called Boson Peak (BP). Looking to the BP and despite its broadness, we can locate the maximum around $v_{B P} \approx 1 \mathrm{THz}$. Values around 1 $\mathrm{THz}$ or $4 \mathrm{meV}$ for the BP in glycerol are in agreement with numerous experimental publications. ${ }^{11,48,62,63}$ Clearly, the excess of modes above the Debye level (or Boson Peak) takes place in the same frequency range that Ioffe-Regel criterion for transverse modes, observation previously reported in other glass-forming systems. ${ }^{9,10,14,64,65}$

In Figure 7, we plot the frequency-dependence of the Brillouin linewidth and velocity of acoustic-like excitations for both polarizations. Looking to the top left graph, showing the damping of the longitudinal waves with frequency in loglog scale, we can see that modes are following a power law with exponent 2 for the temperatures of 350,310 , and $250 \mathrm{~K}$. For these temperatures a relation $\Gamma \propto v^{2}$ is fulfilled for all frequencies in this range. Such a relation is expected in the case of wave propagation in an isotropic medium and, due to our previous observations, seems to be justified for low frequencies. Surprisingly this relation holds until high frequencies, hence beyond the IR frequency delimiting for L modes the propagative to non-propagative nature of vibrational excitations. We must recall here to the reader that in fact a purely $\Gamma \propto v^{2}$ relation is also expected at high frequencies arising from the structural disorder in glasses. ${ }^{58,66,67}$ In definitive, for longitudinal acoustic like excitations in supercooled and liquid state, the damping is related to the mode frequency following a quadratic law. This situation is clearly not the case for L mode in glassy state, where the damping parameter still follows a quadratic law at high frequency but changes to a $v^{3}$ power law for frequencies smaller than $1 \mathrm{THz}$.

Looking to the phase velocity of the longitudinal modes (Figure 7, left bottom), we observe a nearly constant phase velocity at 350,310 , and $250 \mathrm{~K}$ for frequency range greater than $0.6 \mathrm{THz}$ and a small decrease of the phase velocity for lower frequencies. The longitudinal velocities at high frequencies found in our simulations are in excellent agreement with the unrelaxed sound values $c_{\infty}$ found by Brillouin light scattering experiments, ${ }^{32}$ however the low frequency velocities found in our work are incompatible with the relaxed sound of the same reference. This suggests that at these high frequencies we do not probe the relaxation occurring in the longitudinal modes at lower frequencies. At $150 \mathrm{~K}$, we observe that $\mathrm{L}$ velocity shows a slight negative dispersion until $0.9-1 \mathrm{THz}$ followed by an almost flat dispersion towards higher frequencies.

Concerning the $\mathrm{T}$ waves, for all temperatures studied, in high frequency domain (above $1 \mathrm{THz}$ ) we find a relation $\Gamma \propto v^{2}$, in the same manner as for $\mathrm{L}$ modes. Such a relation at high frequencies can still be explained in the case of $\mathrm{T}$ modes by the structural disorder of glasses. In counterpart, different power laws in damping can be visible at low frequencies. At $350 \mathrm{~K}$ and $310 \mathrm{~K}$ (liquid state), we find a 

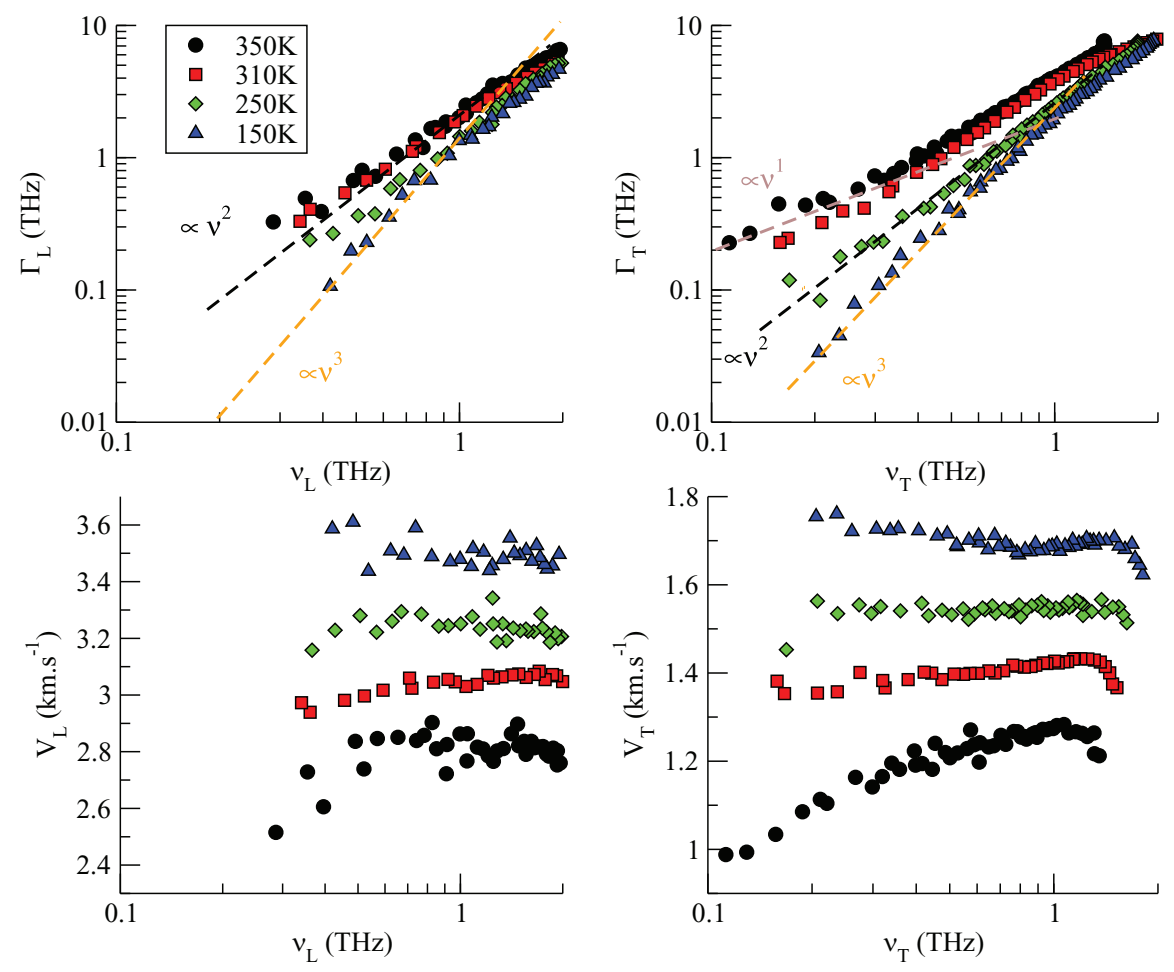

FIG. 7. Frequency dependence of broadening (top) and phase velocity (bottom) of the longitudinal (left) and transverse (right) acoustic-like excitations in glycerol at different studied temperatures. Black circles: $350 \mathrm{~K}$, Red squares: $310 \mathrm{~K}$, Green diamonds: $250 \mathrm{~K}$, and Blue triangles: $150 \mathrm{~K}$. Dashed lines indicate different power laws.

linear law below $0.4 \mathrm{THz}$, confirming our previous observations of a strict relation $v(Q)=\frac{\Gamma(Q)}{2}$ for low wavevectors. The situation is rather different in supercooled state $(250 \mathrm{~K})$, where the aforementioned quadratic relation between damping and frequency is valid, and no change in power law can be distinguished in the frequency range studied. Finally, in glassy state $(150 \mathrm{~K})$, the damping changes from a quadratic law above $1 \mathrm{THz}$ to a $\Gamma \propto \nu^{3}$ power law in low frequency part (below $1 \mathrm{THz}$ ). Hence a clear change in a power law appears in $\mathrm{T}$ modes. Looking to the velocity evolution of $\mathrm{T}$ modes (Figure 7, right bottom), clear change of behavior appears. In liquid state, the transverse velocity is increasing for frequencies up to $0.5 \mathrm{THz}$ and remains nearly constant in the range between $0.5 \mathrm{THz}$ and $1.2 \mathrm{THz}$. After this point the velocity decreases because of the bending of dispersion curve near the pseudo Brillouin zone. At $250 \mathrm{~K}$, T velocity is constant in all the frequency range studied. Finally in glassy state, below $1 \mathrm{THz}$, velocities of $\mathrm{T}$ modes decrease when frequency increases and after $1 \mathrm{THz}$ increase again with frequencies. Invoking a simple viscoelastic model for liquids states permits to understand the increase of phase velocity as the presence of a relaxation process owning a typical frequency of the same magnitude as acoustic excitation. In the same time, the decrease of phase velocity at very high frequency is linked to the bending of the dispersion curve near the pseudo-Brillouin zone. The picture seems more complex in glassy state, where a decrease in phase velocity is observed for frequencies far from the pseudo-Brillouin zone. We would emphasize that all our observations at $150 \mathrm{~K}$ are in agreement with the experiments of Monaco and Giordano ${ }^{11}$ realized on glycerol at the same temperature and seems to be general for glassy state of H-Bonds glass-formers. ${ }^{12}$ In Ref. 11 a change in the $\mathrm{L}$ damping was find for wavevector of $0.22 \AA^{-1}$ from a $Q^{4}$ to a $Q^{2}$ power law and the wavevector quartic dependence of damping was associated to a Rayleigh dispersion of acoustic modes. Rayleigh dispersion appears when an inhomogeneous medium is supporting propagating waves, giving an elastic scattering such as $\Gamma \propto \nu^{4}$ (Ref. 68) or considering a linear relation between velocity and $\mathrm{Q}, \Gamma \propto Q^{4}$. We recall here that more generally in the effective medium approximation, for a percolating network, the Rayleigh law becomes $\Gamma \propto v^{d+1}$ where $d$ is the Euclidean dimensionality. ${ }^{69,70}$ Considering the crossover in longitudinal damping occurring at $0.22 \AA^{-1}$ found by Monaco et al., it remains fully comparable to our findings of a cross-over in damping at frequency close to $1 \mathrm{THz}$ (see Figure 6 for $q-v$ correspondence). In the same time, the value of damping itself falls in fair agreement with our values. The only notable discrepancy arises from the power law found for the longitudinal damping at low frequency, hence considering a linear behavior of frequency with $\mathrm{Q}$, a pure Rayleigh law $\left(\Gamma \propto Q^{4}\right)$ is found at low $\mathrm{Q}$. In our work we are not following a pure Rayleigh dispersion law considering $\Gamma \propto \nu^{3}$ relationship. This apparent discrepancy vanishes considering the nonlinear relationship between $v$ and $\mathrm{Q}$ at low wavevector (attested by the velocity decrease at low frequencies). The nonlinear relation between $v$ and $\mathrm{Q}$, gives an excellent agreement with a quartic power law. In the case of $\mathrm{T}$ modes, considering a linear dependence of damping with frequency in liquid state and a quasi-Rayleigh law in glassy state, at intermediate temperatures and in particular for supercooled states, the power-law must change progressively from 1 to 3 with temperature decrease and hence a quadratic power 
law remains possible at $250 \mathrm{~K}$. Nevertheless, in glassy state we observe a steep increase of damping coupled to a negative dispersion in phase velocities for both $\mathrm{L}$ and $\mathrm{T}$ modes. Furthermore, the minimum in phase velocity happens at the exact location of the BP and clearly indicates a breakdown of the Debye approximation at these time and length scales. We also want to point out the coincidence of the frequency at which the cross-over and minimum of the velocity arise and the location of the BP (around $0.9-1 \mathrm{THz}$ ) and, as stated before, the coincidence of the BP and IR criterion in transverse modes.

\section{DISCUSSION}

Considering on the one hand the wavevector values of the different IR criterion $\left(0.36 \AA^{-1}\right.$ at $150 \mathrm{~K}$ and $0.22 \AA^{-1}$ at $350 \mathrm{~K})$ and on the other hand the relation between the wavelength of the mode and the associated mean-free path $(\lambda / 2 \approx \ell)$, it follows that the relation between wavevector and mean free-path is given by $Q^{I R}=2 \pi / \lambda \approx \pi / \ell$. Applying this relation to the aforementioned IR criterion gives a mean free path of acoustic vibrations between $\ell \approx 14-9 \AA$ which depends on the temperature. It is tempting to relate this mean-free path found in both transverse and longitudinal IR criterion to a structural origin, in particular, recalling that the partial structure factor shows a shoulder at these particular length. In fact our observations and primary conclusions are in agreement with simulations of LJ glass with interconnected isocahedral order. ${ }^{71,72}$ In this work, the mesoscopic order induces a pre-peak in structure factor, pre-peak located around half the First Sharp Diffraction Peak $\left(Q_{p p}\right.$ $\approx Q_{p} / 2$ ). This medium range order in turn deeply affects the transverse and longitudinal acoustic branches for wavevectors in the range of $0.2 Q_{p}$. Said differently, the introduction of a medium range order of characteristic length $\xi$ induces a pre-peak in structural order around $Q_{p p} \approx 2 \pi / \xi$ and a pseudoBrillouin zone at wavevector of roughly $Q_{P B Z} \approx \pi / \xi$ (which does not necessarily commensurate with the pseudo-Brillouin zone given by the local order). In the case of glycerol, the location of the pre-peak (around $0.6-0.7 \AA^{-1}$ ) is globally compatible with the different IR criterion seen between 0.22 and $0.35 \AA^{-1}$. In the case of glassy state, the coincidence between three distinct features (Boson Peak, IR criterion, and strong scattering process) may in fact be rationalized introducing a characteristic size of the heterogeneities. For the lowest frequencies studied, a strong damping process is present which can be understood in terms of Rayleigh elastic scattering on structural heterogeneities of nanometer size and consequently there is no formal reason for the Debye model to hold in this case. We note that, as stated in different works, ${ }^{9,69,70}$ the negative dispersion of sound velocity occurring in glassy state is directly related to the variation of the power-law in damping via Kramers-Kronig relation and is not a manifestation of mode softening. Rayleigh regime will have to cease when half the wavelength will be smaller than the characteristic size of structural inhomogeneities, which in turn corresponds to the IR criterion ${ }^{69}$ and the crossover from strong to weak scattering regime. ${ }^{12,73,74}$ However, the negative dispersion of transverse and longitudinal velocities at increasing frequency will directly modify the DOS. Even taking a Debye approx- imation, the DOS is inversely proportional to $v_{L}^{3}+2 v_{T}^{3}$ and hence a velocity decrease will manifests as a peak in DOS, giving an excess of modes over the Debye model considering a monotonic velocity. ${ }^{9,10}$ The situation is even worse taking a full expression of DOS. ${ }^{9}$ In this case, the mode damping evolution will also contribute to the excess of mode above the Debye level. Following this idea, as longitudinal and transverse modes reach an IR criterion at different frequencies, the previous picture does not explain the location of the BP with the IR criterion in transverse modes only. Two reasons might be invoked here, the first one is the natural degeneracy of the transverse modes, twofold the longitudinal one. A second simple explanation may arise from the prominent contribution of the low frequency modes in reduced DOS. Then as shear waves are slower than compression waves, the latter have a smaller contribution in reduced DOS. Focusing on the liquid state, from an acoustic point of view, the system behaves as a liquid for wavevector less than $0.22-0.28 \AA^{-1}$, limit lying in close vicinity of a second pseudo-Brillouin zone defined by the medium range order location in reciprocal space. In the same time, the analysis of density autocorrelation indicates that a pronounced collective relaxation occurs around this wavevector. Based on these both observations, the collective relaxation must be connected to a limit between a diffusive process at low wavevectors to a non-diffusive process towards higher wavevectors. Previous works on self-dynamics of glycerol have shown a marked cross-over between two regimes around $0.5-0.6 \AA^{-1} \cdot{ }^{19,20}$ Below this wavevector limit, the dynamics is mainly dominated by the standard Fick diffusion whereas for greater wavevectors, the dynamics is dominated by the structural relaxation, observations in agreement with our analysis and recent works tempting to cover the properties of density fluctuations between "local regime" and the continuum limit in glass-forming systems. ${ }^{16,17,21,75,76}$ All these works underline the critical role of mesoscopic order connected to dynamical heterogeneities, which are expected to grow when temperature decreases, ${ }^{17,18,77-79}$ observations in contradiction with our work, where the heterogeneities probed are slightly decreasing with temperature. In our work, we are probing heterogeneities of structural nature (as seen by the $O H$ partial structure factor) which are not necessarily connected to dynamical heterogeneities. ${ }^{80,81}$ Thereby even if some acoustic behaviors on glycerol intermediate glass former are shared among fragile ${ }^{9,10,12}$ and strong glass formers, ${ }^{13,73,74}$ a direct comparison with systems where dynamical heterogeneities play a crucial role in the mesoscopic order remains hazardous. Future works are planned in order to disentangle the effects of structural and dynamical heterogeneities in supercooled glycerol.

\section{CONCLUSIONS}

In conclusion, in the case of glycerol, we can detect the presence of a structural order of mesoscopic size intimately connected to the hydrogen-bond network extension. It appears that medium range order has a collective lifetime comparable to the collective structural relaxation and may be related to the cross-over between structural relaxation and Fick diffusion observed when studying self or collective 
dynamics. In the same time, this structural mesoscopic organization seems to be related to different anomalies detected in acoustic modes (comprising the Boson Peak) for glassy, supercooled, and liquid states simply by considering that meanfree path of the acoustic waves is conditioned by the spatial extent of the mesoscopic order in liquid and supercooled states. Finally, we have to emphasize that even if our hypotheses are strongly supported by our simulations, coupled to a review of experimental literature, we do not pretend to be general and we do not claim that the picture drawn here can be directly applied out of the case of glycerol glass-former.

\section{ACKNOWLEDGMENTS}

This work was supported by ANR Grant PlusDil (ANR12-BS09-0031-01) and Région Pays de La Loire.

${ }^{1}$ M. D. Ediger and P. Harrowell, J. Chem. Phys. 137, 080901 (2012).

${ }^{2}$ S. A. Kivelson and G. Tarjus, Nat. Mater. 7, 831 (2008).

${ }^{3}$ G. Adam and J. H. Gibbs, J. Chem. Phys. 43, 139 (1965).

${ }^{4}$ T. Pezeril, C. Klieber, S. Andrieu, and K. A. Nelson, Phys. Rev. Lett. 102, 107402 (2009).

${ }^{5}$ C. Klieber, T. Pezeril, S. Andrieu, and K. A. Nelson, J. Appl. Phys. 112, 013502 (2012).

${ }^{6}$ C. Klieber, T. Hecksher, T. Pezeril, D. H. Torchinsky, J. C. Dyre, and K. A. Nelson, J. Chem. Phys. 138, 12A544 (2013).

${ }^{7}$ B. Ruzicka, T. Scopigno, S. Caponi, A. Fontana, O. Pilla, P. Giura, G. Monaco, E. Pontecorvo, G. Ruocco, and F. Sette, Phys. Rev. B 69, 100201 (2004).

${ }^{8}$ T. Scopigno, E. Pontecorvo, R. D. Leonardo, M. Krisch, G. Monaco, G. Ruocco, B. Ruzicka, and F. Sette, Philos. Mag. 84, 1453 (2004).

${ }^{9}$ A. Marruzzo, W. Schirmacher, A. Fratalocchi, and G. Ruocco, Sci. Rep. 3, 1407 (2013).

${ }^{10}$ G. Monaco and S. Mossa, Proc. Natl. Acad. Sci. U. S. A. 106, 16907 (2009).

${ }^{11}$ G. Monaco and V. M. Giordano, Proc. Natl. Acad. Sci. U. S. A. 106, 3659 (2009).

${ }^{12}$ B. Ruta, G. Baldi, F. Scarponi, D. Fioretto, V. M. Giordano, and G. Monaco, J. Chem. Phys. 137, 214502 (2012).

${ }^{13}$ C. Ferrante, E. Pontecorvo, G. Cerullo, A. Chiasera, G. Ruocco, W. Schirmacher, and T. Scopigno, Nat. Commun. 4, 1793 (2013).

${ }^{14}$ A. I. Chumakov, G. Monaco, A. Monaco, W. A. Crichton, A. Bosak, R. Rüffer, A. Meyer, F. Kargl, L. Comez, D. Fioretto, H. Giefers, S. Roitsch, G. Wortmann, M. H. Manghnani, A. Hushur, Q. Williams, J. Balogh, K. Parliński, P. Jochym, and P. Piekarz, Phys. Rev. Lett. 106, 225501 (2011).

${ }^{15}$ B. Rufflé, G. Guimbretière, E. Courtens, R. Vacher, and G. Monaco, Phys. Rev. Lett. 96, 045502 (2006).

${ }^{16}$ V. N. Novikov, K. S. Schweizer, and A. P. Sokolov, J. Chem. Phys. 138, 164508 (2013).

${ }^{17}$ A. Furukawa and H. Tanaka, Phys. Rev. Lett. 103, 135703 (2009).

${ }^{18}$ A. Furukawa and H. Tanaka, Phys. Rev. E 84, 061503 (2011).

${ }^{19}$ J. Wuttke, I. Chang, O. G. Randl, F. Fujara, and W. Petry, Phys. Rev. E 54, 5364 (1996).

${ }^{20}$ R. Busselez, R. Lefort, A. Ghoufi, B. Beuneu, B. Frick, F. Affouard, and D. Morineau, J. Phys. Condens. Matter 23, 505102 (2011).

${ }^{21}$ L. Hong, P. D. Gujrati, V. N. Novikov, and A. P. Sokolov, J. Chem. Phys. 131, 194511 (2009).

${ }^{22}$ K. S. Schweizer and E. J. Saltzman, J. Phys. Chem. B 108, 19729 (2004).

${ }^{23}$ J. Colmenero, F. Alvarez, Y. Khairy, and A. Arbe, J. Chem. Phys. 139, 044906 (2013).

${ }^{24}$ U. Bengtzelius, W. Gotze, and A. Sjolander, J. Phys. C: Solid State Phys. 17, 5915 (1984).

${ }^{25}$ J. Blieck, F. Affouard, P. Bordat, A. Lerbret, and M. Descamps, Chem. Phys. 317, 253 (2005).

${ }^{26}$ R. Chelli, P. Procacci, G. Cardini, and S. Califano, Phys. Chem. Chem. Phys. 1, 879 (1999).

${ }^{27}$ R. Chelli, P. Procacci, G. Cardini, R. G. Della Valle, and S. Califano, Phys. Chem. Chem. Phys. 1, 871 (1999).

${ }^{28}$ R. Busselez, R. Lefort, Q. Ji, F. Affouard, and D. Morineau, Phys. Chem. Chem. Phys. 11, 11127 (2009).

${ }^{29}$ S. Plimpton, J. Comput. Phys. 117, 1 (1995).
${ }^{30} \mathrm{R}$. Hockney and J. Eastwood, Computer simulation using particles (Taylor \& Francis, Inc., 1988).

${ }^{31}$ M. Wolfe and J. Jonas, J. Chem. Phys. 71, 3252 (1979).

${ }^{32}$ L. Comez, D. Fioretto, F. Scarponi, and G. Monaco, J. Chem. Phys. 119, 6032 (2003).

${ }^{33}$ J. Powles, Adv. Phys. 22, 1 (1973).

${ }^{34}$ S. R. Elliott, Nature 354, 445 (1991).

${ }^{35}$ D. Morineau and C. Alba-Simionesco, J. Chem. Phys. 109, 8494 (1998).

${ }^{36}$ D. Morineau, C. Alba-Simionesco, M.-C. Bellissent-Funel, and M.-F. Lauthié, Europhys. Lett. 43, 195 (1998).

${ }^{37}$ A. R. Abdel Hamid, R. Lefort, Y. Lechaux, A. Moréac, A. Ghoufi, C. AlbaSimionesco, and D. Morineau, J. Phys. Chem. B 117, 10221 (2013).

${ }^{38}$ A. Hédoux, Y. Guinet, L. Paccou, P. Derollez, and F. Danède, J. Chem. Phys. 138, 214506 (2013).

${ }^{39}$ A. Karmakar, P. Krishna, and R. Joarder, Phys. Lett. A 253, 207 (1999).

${ }^{40}$ A. Perera, F. Sokolić, and L. Zoranić, Phys. Rev. E 75, 060502 (2007).

${ }^{41}$ L. Zoranić, F. Sokolić, and A. Perera, J. Chem. Phys. 127, 024502 (2007).

${ }^{42}$ D. Champeney, R. Joarder, and J. Dore, Mol. Phys. 58, 337 (1986).

${ }^{43}$ M. Garawi, J. Dore, and D. Champeney, Mol. Phys. 62, 475 (1987).

${ }^{44}$ J. J. Towey, A. K. Soper, and L. Dougan, Phys. Chem. Chem. Phys. 13, 9397 (2011).

${ }^{45}$ H.-F. Yuan, T. Xia, M. Plazanet, B. Demé, and M. Orrit, J. Chem. Phys. 136, 041102 (2012).

${ }^{46}$ R. Chelli, F. L. Gervasio, C. Gellini, P. Procacci, G. Cardini, and V. Schettino, J. Phys. Chem. A 104, 5351 (2000).

${ }^{47}$ P. Sillrén, J. Swenson, J. Mattsson, D. Bowron, and A. Matic, J. Chem. Phys. 138, 214501 (2013).

${ }^{48}$ J. Wuttke, W. Petry, G. Coddens, and F. Fujara, Phys. Rev. E 52, 4026 (1995).

${ }^{49}$ R. Busselez, R. Lefort, M. Guendouz, B. Frick, O. Merdrignac-Conanec, and D. Morineau, J. Chem. Phys. 130, 214502 (2009).

${ }^{50}$ J. Wuttke, W. Petry, and S. Pouget, J. Chem. Phys. 105, 5177 (1996).

${ }^{51}$ K. Sköld, Phys. Rev. Lett. 19, 1023 (1967).

${ }^{52}$ P. D. Gennes, Physica 25, 825 (1959).

${ }^{53}$ G. H. Vineyard, Phys. Rev. 110, 999 (1958).

${ }^{54}$ D. Bertolini and A. Tani, Mol. Phys. 75, 1047 (1992).

${ }^{55}$ M. Sampoli, G. Ruocco, and F. Sette, Phys. Rev. Lett. 79, 1678 (1997).

${ }^{56}$ E. Courtens, M. Foret, B. Hehlen, and R. Vacher, Solid State Commun. 117, 187 (2001).

${ }^{57}$ S. N. Taraskin and S. R. Elliott, Phys. Rev. B 61, 12031 (2000).

${ }^{58}$ S. N. Taraskin and S. R. Elliott, J. Phys. Condens. Matter 11, A219 (1999).

${ }^{59}$ H. R. Schober, J. Phys. Condens. Matter 16, S2659 (2004).

${ }^{60}$ E. Courtens, M. Foret, B. Hehlen, B. Rufflé, and R. Vacher, J. Phys. Condens. Matter 15, S1279 (2003).

${ }^{61}$ S. N. Taraskin and S. R. Elliott, Phys. Rev. B 61, 12017 (2000).

${ }^{62}$ S. Kojima, Phys. Rev. B 47, 2924 (1993).

${ }^{63}$ O. Yamamuro, K. Harabe, T. Matsuo, K. Takeda, I. Tsukushi, and T. Kanaya, J. Phys. Condens. Matter 12, 5143 (2000).

${ }^{64}$ H. Shintani and H. Tanaka, Nat. Mater. 7, 870 (2008).

${ }^{65}$ R. Zorn, Physics 4, 44 (2011).

${ }^{66}$ G. Ruocco, F. Sette, R. Di Leonardo, G. Monaco, M. Sampoli, T. Scopigno, and G. Viliani, Phys. Rev. Lett. 84, 5788 (2000).

${ }^{67}$ J. Horbach, W. Kob, and K. Binder, Eur. Phys. J. B 19, 531 (2001).

${ }^{68}$ Lord Rayleigh, Philos. Mag. 47, 375 (1899).

${ }^{69}$ G. Polatsek and O. Entin-Wohlman, Phys. Rev. B 37, 7726 (1988).

${ }^{70}$ C. Ganter and W. Schirmacher, Phys. Rev. B 82, 094205 (2010).

${ }^{71}$ S. Simdyankin, M. Dzugutov, S. Taraskin, and S. Elliott, J. Non-Cryst. Solids 293-295, 327 (2001).

${ }^{72}$ S. I. Simdyankin, M. Dzugutov, S. N. Taraskin, and S. R. Elliott, Phys. Rev. B 63, 184301 (2001).

${ }^{73}$ G. Baldi, M. Zanatta, E. Gilioli, V. Milman, K. Refson, B. Wehinger, B. Winkler, A. Fontana, and G. Monaco, Phys. Rev. Lett. 110, 185503 (2013).

${ }^{74}$ G. Baldi, V. M. Giordano, G. Monaco, and B. Ruta, Phys. Rev. Lett. 104, 195501 (2010).

${ }^{75}$ H. Mizuno and R. Yamamoto, Phys. Rev. Lett. 110, 095901 (2013).

${ }^{76}$ A. Furukawa, Phys. Rev. E 87, 062321 (2013).

${ }^{77}$ C. Dalle-Ferrier, C. Thibierge, C. Alba-Simionesco, L. Berthier, G. Biroli, J.-P. Bouchaud, F. Ladieu, D. L'Hôte, and G. Tarjus, Phys. Rev. E 76, 041510 (2007).

${ }^{78}$ D. Fragiadakis, R. Casalini, and C. M. Roland, Phys. Rev. E 84, 042501 (2011).

${ }^{79}$ K. Kim and S. Saito, J. Chem. Phys. 138, 12 A506 (2013).

${ }^{80}$ A. P. Sokolov, AIP Conf. Proc. 1518, 104 (2013).

${ }^{81}$ W. Kob, S. Roldan-Vargas, and L. Berthier, Nat. Phys. 8, 164 (2012). 